\title{
Comparison of two methods for analysis of gene-environment interactions in longitudinal family data: the Framingham heart study
}

\section{Yun Ju Sung ${ }^{*}{ }^{\dagger}$, Jeannette Simino ${ }^{\dagger}$, Rezart Kume, Jacob Basson, Karen Schwander and D. C. Rao}

Division of Biostatistics, Washington University School of Medicine in St. Louis, St. Louis, MO, USA

\section{Edited by:}

Xuefeng Wang, Harvard University, USA

\section{Reviewed by:}

Yu-Ping Wang, Tulane University, USA Ashok Ragavendran, Massachusetts General Hospital, USA

\section{*Correspondence:}

Yun Ju Sung, Division of Biostatistics, Washington University School of Medicine, 660 South Euclid Avenue, Campus Box 8067, St. Louis, MO 63110, USA

e-mail: yunju@wubios.wustl.edu

${ }^{\dagger}$ Yun Ju Sung and Jeannette Simino have contributed equally to this work.
Gene-environment interaction (GEI) analysis can potentially enhance gene discovery for common complex traits. However, genome-wide interaction analysis is computationally intensive. Moreover, analysis of longitudinal data in families is much more challenging due to the two sources of correlations arising from longitudinal measurements and family relationships. GWIS of longitudinal family data can be a computational bottleneck. Therefore, we compared two methods for analysis of longitudinal family data: a methodologically sound but computationally demanding method using the Kronecker model (KRC) and a computationally more forgiving method using the hierarchical linear model (HLM). The KRC model uses a Kronecker product of an unstructured matrix for correlations among repeated measures (longitudinal) and a compound symmetry matrix for correlations within families at a given visit. The HLM uses an autoregressive covariance matrix for correlations among repeated measures and a random intercept for familial correlations. We compared the two methods using the longitudinal Framingham heart study (FHS) SHARe data. Specifically, we evaluated SNP-alcohol (amount of alcohol consumption) interaction effects on high density lipoprotein cholesterol (HDLC). Keeping the prohibitive computational burden of $\mathrm{KRC}$ in mind, we limited the analysis to chromosome 16, where preliminary cross-sectional analysis yielded some interesting results. Our first important finding was that the HLM provided very comparable results but was remarkably faster than the KRC, making HLM the method of choice. Our second finding was that longitudinal analysis provided smaller $P$-values, thus leading to more significant results, than cross-sectional analysis. This was particularly pronounced in identifying GEls. We conclude that longitudinal analysis of GEls is more powerful and that the HLM method is an optimal method of choice as compared to the computationally (prohibitively) intensive KRC method.

Keywords: gene-environment interactions, longitudinal family data, Framingham heart study, interactions in family data, HLM, SNP-alcohol interactions

\section{INTRODUCTION}

The advent of genomewide association studies (GWAS) has revolutionized the field by identifying hundreds of common genetic variants associated with many common complex disease traits (http://www.genome.gov). However, an important and sobering observation is that these identified loci have very subtle effects, thus explaining only a small fraction of the heritability of most complex traits (Manolio et al., 2009). It is increasingly recognized that the near-exclusive focus on main effects has become a barrier to the identification of additional genes underlying these disease traits. Greater emphasis is being placed in recent years on gene-environment interaction (GEI) analyses (Aschard et al., 2012). The identification of GEIs is important for many reasons (Le Marchand and Wilkens, 2008; Thomas, 2010). GEIs or more complex pathways involving multiple genes and environments can explain part of the missing heritability (McCarthy and Hirschhorn, 2008; Manolio et al., 2009; Eichler et al., 2010; Visscher etal., 2012). They can further elucidate the biological networks underlying complex disease risk and enable "profiling" of individuals at highest risk for disease.

Longitudinal family studies have desirable properties for identifying GEIs by combining the features of repeated measures and family studies. A conventional longitudinal study involves the repeated evaluation of one or more measurable traits in a series of unrelated individuals. Longitudinal data with interesting applications and examples are extensively described in several books (Hand and Crowder, 1996; Verbeke and Molenberghs, 2000). The repeated measurements help reduce error, increase statistical power, and provide a means to study the pattern and determinants of systematic changes in a phenotype of interest over time. For the analysis of longitudinal data, linear mixed-modeling framework is commonly used either with maximum likelihood estimation (Laird and Ware, 1982) or with generalized estimating equations (GEEs; Zeger et al., 1988; Zeger and Liang, 1992). In contrast, a cross-sectional family study utilizes phenotypic similarities and differences amongst close relatives to disentangle the genetic and 
environmental contributions to the trait under study (Khoury and James, 1993). A longitudinal family study further increases the power to resolve the genetic and environmental determinants of traits associated with complex diseases and also to study the corresponding determinants of change in such traits over time (Burton et al., 2005).

However, analysis of longitudinal phenotypes in family data is much more challenging due to two sources of correlations: correlations across longitudinal measurements and correlations among related individuals within families. Most approaches for longitudinal family data are computationally demanding when many subjects are involved, particularly when they need to be executed a large number of times as in the analysis of GWAS data (Kerner et al., 2009). Analysis of GEIs in longitudinal family data further increases the computational burden. In this paper, we compared a methodologically sound but computationally demanding method using the Kronecker model (KRC) with an alternative but computationally more forgiving method using the hierarchical linear model (HLM).

\section{MATERIALS AND METHODS STUDY SAMPLE}

In this study, we used the Framingham Heart Study (FHS) SNP Health Association Resource (SHARe) data, as obtained through the database of genotypes and phenotypes (dbGaPs). The FHS is the oldest prospective longitudinal cohort study of cardiovascular risk factors in the USA. To identify the factors that contribute to cardiovascular disease, FHS began in 1948 with the recruitment of an original cohort of 5,209 men and women who were 2862 years of age at entry (Dawber et al., 1951). Clinic examinations took place approximately every 2 years. In 1971, a second generation of study participants, 5,124 children and spouses of children of the original cohort were enrolled (Feinleib et al., 1975). Clinic examinations took place approximately every 4 years. Enrollment of the third generation cohort of 4,095 children of offspring cohort participants began in 2002 (Splansky et al., 2007). This study obtained informed consent from participants and approval from the appropriate institutional review boards.

\section{GENOTYPE DATA}

Genotype data from the FHS SHARe project include approximately 550,000 SNPs that were genotyped using AffymetrixGeneChip ${ }^{\circledR}$ Human Mapping $500 \mathrm{k}$ Array Set and the 50 k Human Gene Focused Panel. Genotyping involved 10,775 samples (some duplicates) from the three generations of participants (including over 900 pedigrees). Genotype calls were made with the BRLMM algorithm. The genotyping data for the 10,043 samples from 9,354 participants that passed the Affymetrix criteria were additionally checked for sex consistency and consistency with family structure, resulting in genotyping data for 9,274 participants in FHS SHARe. More detailed information is available elsewhere (Cupples et al., 2009).

\section{STATISTICAL ANALYSES}

Longitudinal family studies have more complex phenotypic correlation structure than cross-sectional family studies or longitudinal studies of unrelated individuals, as phenotypes are repeatedly measured among related individuals. Repeated measurements for the same individual are temporally correlated. Phenotypes on related individuals at each time are subject to familial correlations due to shared genetic and environmental effects. Furthermore, phenotypes of related individuals at different time points are also correlated due to both factors.

To account for these sources of correlation, we compare two approaches that belong to the mixed modeling framework (Laird and Ware, 1982), one known for its theoretical soundness and the other known for its computational speed. Our first approach models the full variance-covariance of a phenotypic vector as a Kronecker (KRC) product of two variance-covariance matrices: $\Sigma_{\text {visit }} \otimes \Omega_{\text {family }}$, where $\Sigma_{\text {visit }}$ is the covariance matrix across visits and $\Omega_{\text {family }}$ is the covariance matrix across family members. Because this methodology has a desirable property that two sources of correlation independently contribute to the overall covariance structure (Galecki, 1994), the KRC is a methodologically sound approach for analysis of longitudinal family data. However, the KRC belongs to the curved exponential family, which makes estimation and testing even more computationally demanding than the unstructured covariance matrix (Roy, 2008). Our second approach is a three-level HLM. Multilevel models are appropriate for data that are organized at more than one level (i.e., nested data). The HLM is also called a mixed model with nested random effects (Berry et al., 2007). We assume that repeated measurements (at the lowest level 1) are nested within individuals (at the next level 2), who are further clustered within families (at the highest level 3). Due to this nested structure, the HLM is computationally more feasible.

For both KRC and HLM approaches in the analysis of longitudinal family data, we use SAS PROC MIXED (Littell, 2006). For a Kronecker product covariance, SAS provides three options: unstructured with compound symmetry (UN@CS), unstructured with order 1 autoregressive (UN@AR(1)), and unstructured with unstructured (UN@UN). In our KRC analysis, we use an unstructured matrix for longitudinal correlation and compound symmetry matrix for familial correlations. In our HLM approach, we use an autoregressive-moving-average $(1,1)$ covariance matrix for longitudinal correlations and a random intercept for familial correlations(Littell, 2006). When there is no longitudinal data (i.e., a cross-sectional dataset within a single visit), the KRC model with a compound symmetry matrix for familial correlation is mathematically identical to the HLM approach with a random intercept for familial correlations.

To identify GEIs, we consider the following three models for the expected response trait $(Y)$ :

$$
\begin{aligned}
& \text { Model } 1: E[Y]=\alpha+\beta_{\mathrm{c}} C+\beta_{\mathrm{e}} E, \\
& \text { Model } 2: E[Y]=\alpha+\beta_{\mathrm{c}} C+\beta_{\mathrm{e}} E+\beta_{\mathrm{g}} G, \text { and } \\
& \text { Model } 3: E[Y]=\alpha+\beta_{\mathrm{c}} C+\beta_{\mathrm{e}} E+\beta_{\mathrm{g}} G+\beta_{\mathrm{ge}} G E
\end{aligned}
$$

where $\beta_{\mathrm{c}}$ is the common covariate effect, $\beta_{\mathrm{e}}$ is the environmental main effect, $\beta_{\mathrm{g}}$ is the genetic main effect, and $\beta_{\mathrm{ge}}$ is the GEI effect. For both KRC and HLM approaches described 
previously, we obtain maximum likelihood (by running SAS version 9.3 PROC MIXED with METHOD = ML) separately using each of the three mean models. Instead of the Satterthwaite and Kenward-Roger degrees of freedom methods, we use EMPICAL option for the estimated variance-covariance matrix of the fixed-effects parameters by using the asymptotically consistent estimator (Huber, 1967; White, 1980; Liang and Zeger, 1986). To test the genetic main effect $\left(H_{0}: \beta_{g}=0\right)$, we use the likelihood ratio test based on model 1 and 2 , which follows a chi-squared distribution with 1 degrees of freedom (df) under the null hypothesis. To test the GEI effect $\left(H_{0}: \beta_{\mathrm{ge}}=0\right)$, we use the likelihood ratio test based on model 2 and 3 where both models include the genetic main effect. Finally to jointly test genetic main and GEI effects $\left(H_{0}: \beta_{\mathrm{g}}=\beta_{\mathrm{ge}}=0\right)$ proposed by Kraft et al. (2007), we use the likelihood ratio test based on model 1 and 3, which follows a chi-squared distribution with $2 \mathrm{df}$ under the null hypothesis. The SAS code is available upon request.

To compare the two methods of analysis and to further investigate whether longitudinal analysis enhances identification of GEIs, we create a dataset that optimize the use of the "original" cohort that was measured 27 times and the "offspring" cohort that was measured seven times (as of when we obtained the FHS SHARe data from $\mathrm{dbGaP}$ ). We create a dataset that include all seven offspring visits and the corresponding seven visits of the original cohort (matched by the closest visit dates). In addition, for practical reasons related to missing data and time required to complete the analyses, we reduce our dataset to include the most recent five of these seven visits, effectively reducing our dataset to multigeneration families measured at 5-visits. For this investigation, we consider three sets of analyses: 1-visit data (the most recent visit), 3-visit data (by excluding alternate visits), and all 5-visit data. We analyze high density lipoprotein cholesterol (HDLC) concentration in blood serum. We use amount of alcohol (ounces) consumed per week as an interacting covariate. In addition, age, sex, body mass index (BMI), and use of anti-lipid medications are used as common covariates in the analysis of all three datasets described above.

Our analysis is restricted to chromosome 16, where crosssectional analysis based on a single visit yielded significant results. Out of 15,259 SNPs on chromosome 16, we exclude SNPs that have Hardy-Weinberg equilibrium (HWE) $P$-value less than $10^{-6}$, resulting in 14,026 SNPs. HWE $P$-values are computed based on founders only using PLINK (Purcell et al., 2007), as recommended for family studies. Due to a very long computational time, analysis with KRC for the 5-visit data set is further restricted to a subset of 67 SNPs.

\section{RESULTS}

Table 1 presents select characteristics of the longitudinal family data that were used for the analysis. Sample size varied across the three datasets used for the analysis: 3,012 subjects for 1-visit, 3,946 unique subjects for 3-visits and 4,190 unique subjects for 5-visits. Our analysis dealt with partially missing longitudinal phenotypes, as indicated by the varying sample sizes. The characteristics were consistent across the three datasets. One notable exception was the use of anti-lipid medication, which was $21 \%$ in the 1 -visit dataset and about $9 \%$ in the 3 -visit and 5-visits datasets. Given that the 1 -visit data corresponded to the last visit, this is expected because a medication use in longitudinal studies tends to increase over time.

Results were compared between Kronecker (KRC) and HLM approaches for all three hypothesis tests ( $1 \mathrm{df}$ genetic main effect, $1 \mathrm{df}$ interaction effect in the presence of the genetic main effect, and $2 \mathrm{df}$ joint tests of main and interaction effects) and also for all three data sets. In the 1-visit data, we empirically confirmed that our KRC model provided identical results as our HLM approach for all three hypothesis tests. As shown in Figure 1, the results $[-\log (P)$ values] between KRC and HLM approaches correlated very well for all three tests and for both 3-visit and 5-visit data, providing correlation of over 0.96 in all 6-cases.

Our analysis was run using a cluster of multiple Linux computers. However, to compare CPU time of the two approaches in the three datasets, a subset of analysis with the two approaches was repeated using the identical Linux computer. CPU time with the HLM approach was averaged over 1,500 SNPs for all three datasets. CPU time with the KRC approach was averaged over 1,500, 77, and 6 SNPs for 1-, 3-, and 5-visit data, respectively. As shown in Table 2, analysis with the HLM approach was faster than analysis with the KRC approach for all three datasets. This advantage in CPU time was increasingly more pronounced as the data included more visits. Under the identical Linux computer, HLM was 85 times faster

Table 1 | Descriptive statistics of the longitudinal family data used in the analysis.

\begin{tabular}{llll}
\hline Characteristics & 1-visit & 3-visits & 5-visits \\
\hline Unique individuals, $n$ & 3,012 & 3,946 & 4,190 \\
Observed data, $n$ & 3,012 & 9,620 & 16,480 \\
Male, $n$ (\%) & $1,397(46.38 \%)$ & $4,496(46.74 \%)$ & $7,703(46.74 \%)$ \\
Anti-lipids med use, $n(\%)$ & $624(20.72 \%)$ & $921(9.57 \%)$ & $1,462(8.87 \%)$ \\
Age, years & $60.75 \pm 9.25$ & $55.53 \pm 11.68$ & $55.89 \pm 11.54$ \\
BMl, kg/m² & $28.16 \pm 5.31$ & $27.26 \pm 5.01$ & $27.28 \pm 4.99$ \\
Alcohol, oz./week & $2.61 \pm 3.79$ & $2.81 \pm 4.11$ & $2.73 \pm 4.06$ \\
HDLC, $\mathrm{mg} / \mathrm{dL}$ & $53.74 \pm 17.05$ & $51.57 \pm 15.75$ & $51.05 \pm 15.65$
\end{tabular}



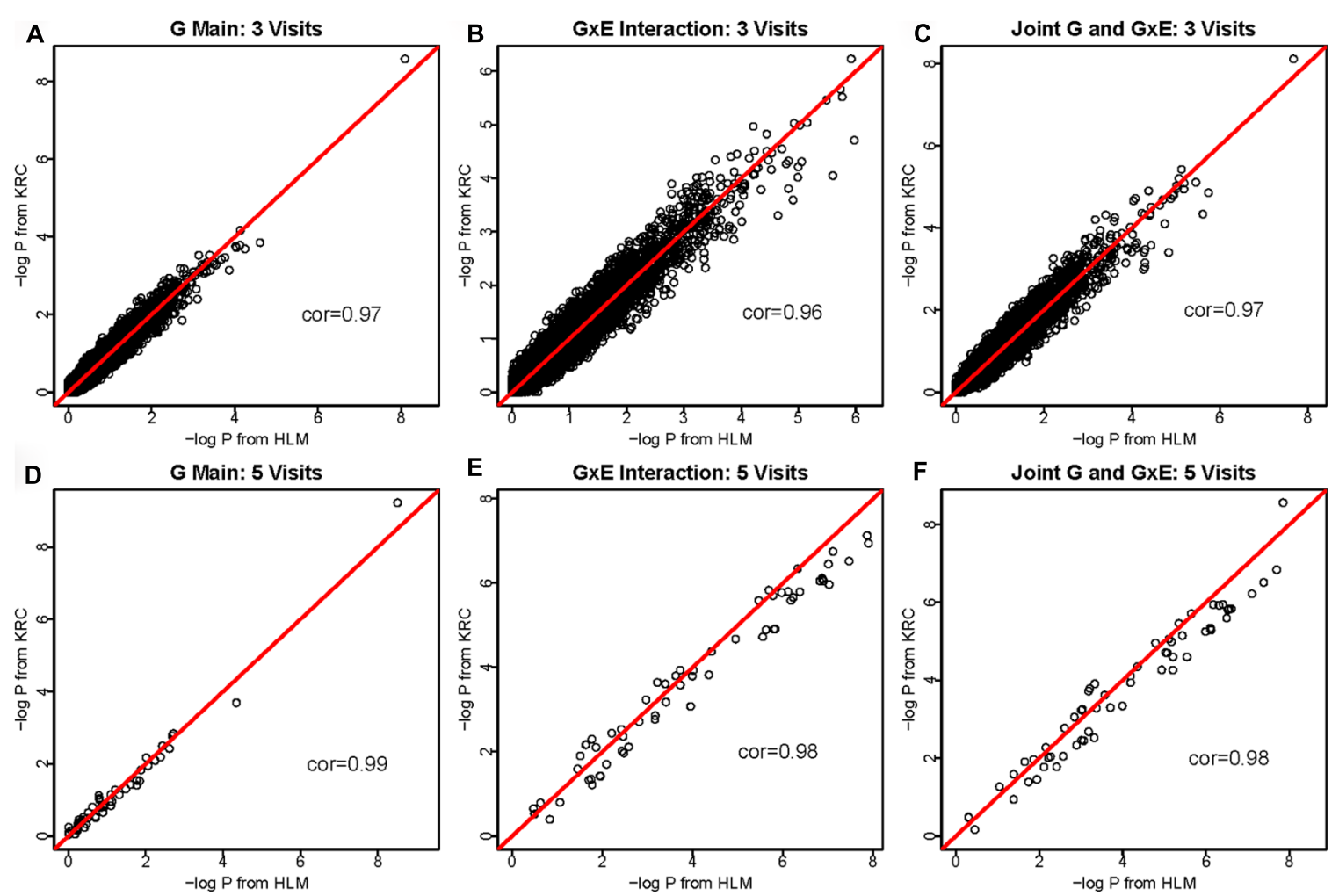

FIGURE $1 \mid-\log (P)$ values using the HLM ( $x$-axis) versus $-\log (P)$ values using the KRC ( $y$-axis) for 3 -visit and 5-visit data sets.

Results from both methods were identical for 1-visit data (not shown). The 1st row corresponds to the 3-visit results (A for genetic main effects, B for GEl effects, and $\mathbf{C}$ for joint 2 df genetic and GEl effects). Similarly, the 2nd row corresponds to the 5-visits results (D for genetic main effects, $\mathbf{E}$ for GEl effects, and $\mathbf{F}$ for joint 2 df genetic and GEl effects). for the 3-visit data (1.42 sec vs. 1.43 min per SNP) and 1,000 times faster for the 5 -visit data ( $2.5 \mathrm{sec}$ vs. $42.3 \mathrm{~min}$ per SNP).

To investigate whether longitudinal analysis enhances statistical significance, we examined the $-\log (P)$ values using longitudinal data (with 3-visit and 5-visit) and those using one visit only. We found that either longitudinal analysis (3-visit or 5-visit) provided smaller $P$-values, thus leading to more significant results, than the cross-sectional analysis (with 1-visit). In particular, as shown in Table 3, the gain in statistical significance was particularly pronounced for identifying GEIs. For example, the number of SNPs with $P$-value $<1 \times 10^{-5}$ for identifying GEIs was $8,10,31$ from 1-, 3-, and 5-visit data, respectively. Similar pattern was also found for jointly identifying the main and interaction effects. Figure 2 shows results from the 1-visit data set and those from the 5-visit data set. In Figure 2, more SNPs are above the diagonal line: 53, 54, and $55 \%$ of SNPs had smaller $P$-values from the 5-visit data for identifying main effect, interaction effect, and joint effects, respectively. Similar pattern was also found between results from the 3-visit data and those from the 1-visit data (not shown; available upon request).

\section{DISCUSSION}

Complex traits such as blood pressure and cholesterol vary with age and depend on both genetic and environmental factors. For such traits, longitudinal studies are valuable to disentangle genetic and environmental effects. Longitudinal studies also enable the prospective measurement of time-varying factors that are not included in typical genetic studies. The use of longitudinal data in studies of GEIs is particularly appealing because some of the power lost in the analysis of GEIs may be recaptured by the use of longitudinal data. As time-varying covariates are measured with improved precision in longitudinal studies, longitudinal data analysis can enhance the identification of GEIs. With additional features of family studies that utilize phenotypic similarities and differences among relatives to disentangle the genetic and environmental risk factors, longitudinal family studies can further increase the power to resolve the genetic and environmental determinants of traits associated with complex diseases.

Table 2 | CPU time for running analysis with three mean models at each SNP.

\begin{tabular}{llll}
\hline Model & 1-visit & 3-visits & 5-visits \\
\hline Kronecker model & $0.55 \mathrm{~s}$ & $1.43 \mathrm{~m}$ & $42.33 \mathrm{~m}$ \\
Hierarchical linear model & $0.41 \mathrm{~s}$ & $1.42 \mathrm{~s}$ & $2.54 \mathrm{~s}$
\end{tabular}

Hierarchical linear model runs were averaged over 1,500 SNPS. KRC run times were averaged over 1,500, 77, and 6 SNPs for 1, 3, and 5 visits, respectively. 
Table 3 | Number of SNPs with $\boldsymbol{P}$-values below thresholds.

\begin{tabular}{|c|c|c|c|c|c|c|c|c|c|}
\hline & \multicolumn{3}{|c|}{ Genetic main effect } & \multicolumn{3}{|c|}{ Gene-environment interaction effect } & \multicolumn{3}{|c|}{ Joint main and interaction } \\
\hline & 1-visit & 3-visits & 5 visits & 1-visit & 3-visits & 5-visits & 1-visit & 3-visits & 5-visits \\
\hline$P<1 \times 10^{-6}$ & 0 & 1 & 1 & 4 & 0 & 16 & 3 & 1 & 15 \\
\hline$P<1 \times 10^{-7}$ & 0 & 1 & 1 & 0 & 0 & 6 & 0 & 1 & 4 \\
\hline
\end{tabular}
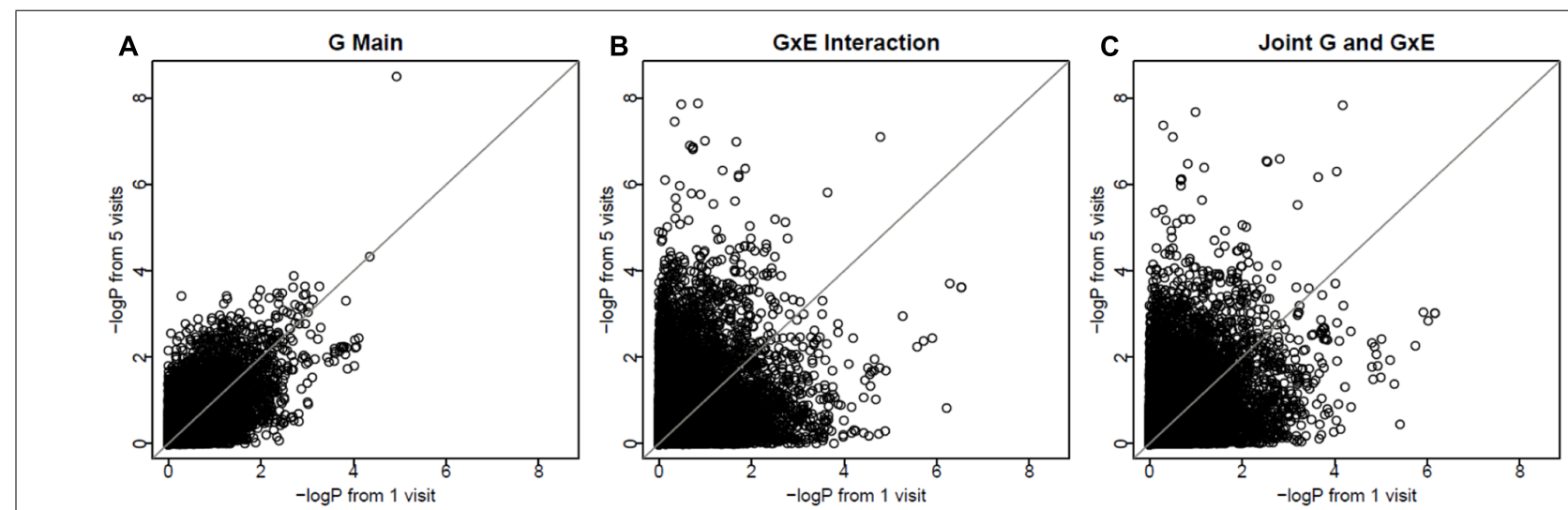

FIGURE 2 | More visits are better for identifying interactions. The $x$-axis shows $-\log (P)$ values using the 1 -visit data set, and the $y$-axis shows $-\log (P)$ values using the 5-visit data set. More SNPs $(53,54$, and 55\% of SNPs in A-C, respectively) are above the diagonal line.

However, analysis of longitudinal family data is known to be more challenging due to two sources of correlations arising from repeated measures and family relationships. One may use two-step modeling, in which the repeated measurements are first reduced to a single summary measure per subject by applying some form of data reduction procedure. For example, either quantitative change in a phenotype between the first and last measurements or a slope from a regression model for each individual can be used as a summary measure (Gee et al., 2003). These summary measures may then be analyzed with a standard method for cross-sectional family data. However, such two-step modeling has been shown to be less efficient than single joint modeling that makes use of all the data simultaneously (Gauderman et al., 2003). Moreover, unlike joint modeling, most two-step modeling approaches fail to properly account for uncertainty in the value of the summary measures when fitting the second step. The linear mixed-effect modeling framework that we used in this paper makes use of all the data simultaneously and therefore is preferable (Laird and Ware, 1982).

In this paper, we evaluated two linear mixed-effect models for analysis of longitudinal family data. We compared $P$-values using two models, as they are the most commonly used measure for the association analysis in GWAS setting. Our $P$-values are based on the likelihood ratio tests for the fixed effects that correspond to the genetic main and GEI effects. As suggested by the reviewer, we note that the mixed-effect models are also widely used in quantitative genetics research (Lynch and Walsh, 1998; Mrode and Thompson, 2005). Both heritability estimation
(Hopper and Mathews, 1982) and variance component analysis (Amos, 1994) in human genetics research are based on a linear mixed-model that includes a random-effect accounting for unobserved polygenic effects. The restricted maximum likelihood (REML) approach is also commonly used for the estimation of variance components (random effect parameters; Patterson and Thompson, 1971). In addition to SAS, packages such as ASREML (Gilmour et al., 1995) and BLUPf90 (Misztal, 2008) can be used for the REML estimation of the random effect parameters. Also using linear mixed model and the REML approach, the GCTA software has been recently developed to estimate trait variation with GWAS data in order to address the "missing heritability" problem (Yang et al., 2011). A more comprehensive investigation using these packages for the GEI analysis of longitudinal family data would be valuable for improving computational efficiency.

There are several limitations in our work. First, the correlation structure of our Kronecker (KRC) modeling was an unstructured matrix for longitudinal correlation and a compound symmetry matrix for familial correlations (UN@CS). A more appropriate model would be order 1 autoregressive for longitudinal correlation and a compound symmetry for familial correlations [AR(1)@CS]. Because SAS does not provide this option, other packages including those described in the previous paragraph may be more flexible to evaluate various models. Second, we used an autoregressivemoving-average $(1,1)$ covariance matrix for longitudinal correlations and a random intercept for familial correlations in our HLM 
approach. We chose this because, under no longitudinal data, the KRC model with a compound symmetry matrix for familial correlation is mathematically identical to the HLM approach with a random intercept for familial correlations. Instead of a random intercept, use of kinship matrix may be a better approach to account for familial correlation. Third, our investigation is entirely practically driven. We implicitly assumed that the KRC model is the appropriate model due to its theoretical soundness and evaluated whether the computationally faster HLM approach provides comparable results. Because of this, model selection criteria such as AIC or BIC have not been used to evaluate the differences in model performances. The "sandwich" estimate option that we used for the variance-covariance matrix of the fixed-effects parameters is known to provide robust and asymptotically consistent estimator under miss-specified models (Huber, 1967; White, 1980; Liang and Zeger, 1986). However, as the reviewer noted, the estimates of fixed effects are still a function of the estimated variance components and may change with the random effects specification.

This study provided empirical findings that longitudinal analysis is more powerful and that the HLM is an optimal method of choice as compared to the computationally more intensive Kronecker (KRC) modeling. Our previous work (Shi et al., 2009) presented a proof of concept that the longitudinal approach using HLM can be more powerful than a cross-sectional analysis. The alternative KRC modeling is computationally very intensive. To the best of our knowledge, this is the first paper that provides systematic comparison between HLM and KRC approaches. Our most important finding was that the HLM provided very comparable results but remarkably faster than the KRC, making HLM the method of choice. Our second finding was that longitudinal analysis provided smaller $P$-values, thus leading to more significant results, than the cross-sectional analysis. The gain in statistical significance from longitudinal analysis may be due to the increased number of (repeated) observations. However, the increased sample size alone does not explain why the significance gain was more pronounced for identifying GEIs. GEIs are more difficult to identify and prone to have large standard errors; this is exactly where longitudinal data appear to be more powerful because the repeated measurements help reduce error, thereby increasing statistical power. As our findings were based on an empirical evaluation using data on a single chromosome, they may not be generalized to all situations. As greater emphasis is being placed in recent years on GEI analyses (Aschard et al., 2012), a more comprehensive investigation would strengthen our findings in this timely topic.

\section{AUTHOR CONTRIBUTIONS}

Yun Ju Sung, Jeannette Simino, and D. C. Rao jointly conceived and designed the study. Yun Ju Sung summarized the results and drafted the manuscript. Jeannette Simino led implementation of the methods in SAS and quality control of the results. Rezart Kume performed all analyses and summarized the results. Jacob Basson carried out quality control of the imputations. Karen Schwander prepared all datasets, performed quality control, and quality controlled the results by independently running a subset of the analyses. D. C. Rao provided administrative support and helped
Yun Ju Sung in summarizing and interpreting the results. All authors have reviewed and approved the final manuscript.

\section{ACKNOWLEDGMENTS}

We thank the two reviewers for their constructive and insightful comments, which substantially improved the manuscript. Our investigation was supported by grant R01 HL107552 from the National Heart, Lung, and Blood Institute (NHLBI). The Framingham Heart Study is conducted and supported by the NHLBI in collaboration with Boston University (contract no. N01-HC25195). Funding for SHAReAffymetrix genotyping was provided by NHLBI contract N02-HL-64278. This manuscript was not prepared in collaboration with investigators of the Framingham Heart Study and does not necessarily reflect the opinions or views of the Framingham Heart Study, Boston University, or NHLBI.

\section{REFERENCES}

Amos, C. I. (1994). Robust variance-components approach for assessing genetic linkage in pedigrees. Am. J. Hum. Genet. 54, 535-543.

Aschard, H., Lutz, S., Maus, B., Duell, E. J., Fingerlin, T. E., Chatterjee, N., et al. (2012). Challenges and opportunities in genome-wide environmental interaction (GWEI) studies. Hum. Genet. 131, 1591-1613. doi: 10.1007/s00439-0121192-0

Berry, S. D., Samelson, E. J., Hannan, M. T., Mclean, R. R., Lu, M., Cupples, L. A., et al. (2007). Second hip fracture in older men and women: the Framingham study. Arch. Intern. Med. 167, 1971-1976. doi: 10.1001/archinte.167.18.1971

Burton, P. R., Scurrah, K. J., Tobin, M. D., and Palmer, L. J. (2005). Covariance components models for longitudinal family data. Int. J. Epidemiol. 34, 1063-1077: discussion 1077-1069. doi:10.1093/ije/dyi069

Cupples, L. A., Heard-Costa, N., Lee, M., Atwood, L. D., and Framingham Heart Study, I. (2009). Genetics analysis workshop 16 problem 2: the Framingham heart study data. BMC Proc. 3(Suppl. 7), S3. doi: 10.1186/1753-6561-3-s7-s3

Dawber, T. R., Meadors, G. F., and Moore, F. E. Jr. (1951). Epidemiological approaches to heart disease: the Framingham study. Am. J. Public Health Nations Health 41, 279-281. doi: 10.2105/AJPH.41.3.279

Eichler, E. E., Flint, J., Gibson, G., Kong, A., Leal, S. M., Moore, J. H., et al. (2010). Missing heritability and strategies for finding the underlying causes of complex disease. Nat. Rev. Genet. 11, 446-450. doi: 10.1038/nrg2809

Feinleib, M., Kannel, W. B., Garrison, R. J., McNamara, P. M., and Castelli, W. P. (1975). The Framingham offspring study. Design and preliminary data. Prev. Med. 4, 518-525. doi: 10.1016/0091-7435(75)90037-7

Galecki, A. T. (1994). General-class of covariance-structures for 2 or more repeated factors in longitudinal data-analysis. Commun. Stat. Theory Methods 23, 31053119. doi: 10.1080/03610929408831436

Gauderman, W. J., Macgregor, S., Briollais, L., Scurrah, K., Tobin, M., Park, T., et al. (2003). Longitudinal data analysis in pedigree studies. Genet. Epidemiol. 25(Suppl. 1), S18-S28. doi: 10.1002/gepi.10280

Gee, C., Morrison, J. L., Thomas, D. C., and Gauderman, W. J. (2003). Segregation and linkage analysis for longitudinal measurements of a quantitative trait. $B M C$ Genet. 4(Suppl. 1), S21. doi:10.1186/1471-2156-4-S1-S21

Gilmour, A. R., Thompson, R., and Cullis, B. R. (1995). Average information REML: an efficient algorithm for variance parameter estimation in linear mixed models. Biometrics 51, 1440-1450. doi: 10.2307/2533274

Hand, D. J., and Crowder, M. J. (1996). Practical Longitudinal Data Analysis. New York: Taylor \& Francis. doi: 10.1007/978-1-4899-3033-0

Hopper, J. L., and Mathews, J. D. (1982). Extensions to multivariate normal models for pedigree analysis. Ann. Hum. Genet. 46, 373-383. doi: 10.1111/j.1469-1809.1982.tb01588.x

Huber, P. J. (1967). The behavior of maximum likelihood estimates under nonstandard conditions. Proc. Fifth Berkeley Symp. Math. Stat. Prob. 1, 221-233.

Kerner, B., North, K. E., and Fallin, M. D. (2009). Use of longitudinal data in genetic studies in the genome-wide association studies era: summary of Group 14. Genet. Epidemiol. 33(Suppl. 1), S93-S98. doi: 10.1002/gepi.20479 
Khoury, M. J., and James, L. M. (1993). Population and familial relative risks of disease-associated with environmental-factors in the presence of geneenvironment interaction. Am. J. Epidemiol. 137, 1241-1250.

Kraft, P., Yen, Y. C., Stram, D. O., Morrison, J., and Gauderman, W. J. (2007). Exploiting gene-environment interaction to detect genetic associations. Hum. Hered. 63, 111-119. doi: 10.1159/000099183

Laird, N. M., and Ware, J. H. (1982). Random-effects models for longitudinal data. Biometrics 38, 963-974. doi: 10.2307/2529876

Le Marchand, L., and Wilkens, L. R. (2008). Design considerations for genomic association studies: importance of gene-environment interactions. Cancer Epidemiol. Biomarkers Prev. 17, 263-267. doi: 10.1158/1055-9965.EPI-07-0402

Liang, K. Y., and Zeger, S. L. (1986). Longitudinal data-analysis using generalized linear-models. Biometrika 73, 13-22. doi: 10.1093/biomet7/3.1.13

Littell, R. C. (2006). SAS for Mixed Models. Cary, NC: SAS Institute.

Lynch, M., and Walsh, B. (1998). Genetics and Analysis of Quantitative Traits. Sunderland, MA: Sinauer Associates, Inc.

Manolio, T. A., Collins, F. S., Cox, N. J., Goldstein, D. B., Hindorff, L. A., Hunter, D. J., et al. (2009). Finding the missing heritability of complex diseases. Nature 461, 747-753. doi: 10.1038/nature08494

McCarthy, M. I., and Hirschhorn, J. N. (2008). Genome-wide association studies: potential next steps on a genetic journey. Hum. Mol. Genet. 17, R156-R165. doi: $10.1093 / \mathrm{hmg} / \mathrm{ddn} 289$

Misztal, I. (2008). Reliable computing in estimation of variance components. J. Anim. Breed Genet. 125, 363-370. doi: 10.1111/j.1439-0388.2008.00774.x

Mrode, R. A., and Thompson, R. (2005). Linear Models for the Prediction of Animal Breeding Values. Cambridge, MA: CABI Publisher. doi: 10.1079/9780851990002.0000

Patterson, H. D., and Thompson, R. (1971). Recovery of inter-block information when block sizes are unequal. Biometrika 58, 545-554. doi 10.1093/biomet/58.3.545

Purcell, S., Neale, B., Todd-Brown, K., Thomas, L., Ferreira, M. A., Bender, D., et al. (2007). PLINK: a tool set for whole-genome association and population-based linkage analyses. Am. J. Hum. Genet. 81, 559-575. doi: 10.1086/519795

Roy, A. (2008). Computation aspects of the parameter estimates of linear mixed effects model in multivariate repeated measures set-up. J. Appl. Stat. 35, 307-320. doi: 10.1080/02664760701833271

Shi, G., Rice, T. K., Gu, C. C., and Rao, D. C. (2009). Application of three-level linear mixed-effects model incorporating geneage interactions for association analysis of longitudinal family data. BMC Proc. 3(Suppl. 7), S89. doi:10.1186/1753-65613-S7-S89
Splansky, G. L., Corey, D., Yang, Q., Atwood, L. D., Cupples, L. A., Benjamin, E. J., et al. (2007). The Third Generation Cohort of the National Heart, Lung, and Blood Institute's Framingham Heart Study: design, recruitment, and initial examination. Am. J. Epidemiol. 165, 1328-1335. doi: 10.1093/aje/kwm021

Thomas, D. (2010). Gene-environment-wide association studies: emerging approaches. Nat. Rev. Genet. 11, 259-272. doi: 10.1038/nrg2764

Verbeke, G., and Molenberghs, G. (2000). Linear Mixed Models for Longitudinal Data. New York: Springer.

Visscher, P. M., Brown, M. A., McCarthy, M. I., and Yang, J. (2012). Five years of GWAS discovery. Am. J. Hum. Genet. 90, 7-24. doi: 10.1016/j.ajhg.2011.11.029

White, H. (1980). A heteroskedasticity-consistent covariance-matrix estimator and a direct test for heteroskedasticity. Econometrica 48, 817-838. doi: 10.2307/ 1912934

Yang, J., Lee, S. H., Goddard, M. E., and Visscher, P. M. (2011). GCTA: a tool for genome-wide complex trait analysis. Am. J. Hum. Genet. 88, 76-82. doi: 10.1016/j.ajhg.2010.11.011

Zeger, S. L., and Liang, K. Y. (1992). An overview of methods for the analysis of longitudinal data. Stat. Med. 11, 1825-1839. doi: 10.1002/sim. 4780111406

Zeger, S. L., Liang, K. Y., and Albert, P. S. (1988). Models for longitudinal data: a generalized estimating equation approach. Biometrics 44, 1049-1060. doi: $10.2307 / 2531734$

Conflict of Interest Statement: The authors declare that the research was conducted in the absence of any commercial or financial relationships that could be construed as a potential conflict of interest.

Received: 30 August 2013; accepted: 09 January 2014; published online: 30 January 2014.

Citation: Sung YJ, Simino J, Kume R, Basson J, Schwander K and Rao DC (2014) Comparison of two methods for analysis of gene-environment interactions in longitudinal family data: the Framingham heart study. Front. Genet. 5:9. doi: 10.3389/fgene.2014.00009

This article was submitted to Statistical Genetics and Methodology, a section of the journal Frontiers in Genetics.

Copyright (c) 2014 Sung, Simino, Kume, Basson, Schwander and Rao. This is an openaccess article distributed under the terms of the Creative Commons Attribution License (CC BY). The use, distribution or reproduction in other forums is permitted, provided the original author(s) or licensor are credited and that the original publication in this journal is cited, in accordance with accepted academic practice. No use, distribution or reproduction is permitted which does not comply with these terms. 